\title{
THE PRODUCT THEOREM FOR TOPOLOGICAL ENTROPY
}

\author{
BY \\ L. WAYNE GOODWYN
}

The purpose of this paper is to prove the following product theorem:

THeOREM 2. Let $X$ and $Y$ be compact Hausdorff spaces and let $T: X \rightarrow X$ and $S: Y \rightarrow Y$ be continuous. Then

$$
h(T \times S)=h(T)+h(S),
$$

where $h$ denotes topological entropy [1], and $T \times S: X \times Y \rightarrow X \times Y$ is defined as

$$
T \times S(x, y)=(T x, S y) \text { for }(x, y) \in X \times Y .
$$

This theorem is stated in [1] without the assumption that $X$ and $Y$ be Hausdorff. However, the first half of the proof depends on the assertion that for open covers $\alpha$ of $X$ and $\beta$ of $Y, N(\alpha \times \beta)=N(\alpha) \cdot N(\beta)$. Without much difficulty one can construct examples where this equality does not hold, so that the proof in [1] yields only that $h(T \times S) \leqq h(T)+h(S)$.

1. Introduction. By a flow we mean a pair $(X, T)$, where $X$ is a compact Hausdorff space and $T: X \rightarrow X$ is a continuous map. Throughout the paper, $(X, T)$ and $(Y, S)$ will denote arbitrary flows. If $T$ is a homeomorphism from $X$ onto $X$, we say that $(X, T)$ is a cascade.

In $\$ 2$ we prove the product theorem for cascades, proving it first for subcascades of sequence cascades. In $\S 3$ we prove an inverse limit theorem which is used in $\S 4$ to generalize the product theorem. In $\$ 5$ we give an example which shows that closed covers can, in general, yield larger entropy than open covers.

We shall use some notation and results from both [1] and [3]. We say that a cover $\beta$ of $X$ is a shrinking of a cover $\alpha$ if there is a function $g$ from a subcollection $\alpha^{\prime}$ of $\alpha$ onto $\beta$ such that

$$
g(A) \subset A \text { for all } A \in \alpha^{\prime} .
$$

It is clear that in this case, the order of $\beta$ is less than or equal to the order of $\alpha$.

We say that a cover is nonoverlapping if the intersection of any two different members has no interior. Note that a shrinking of a nonoverlapping cover is nonoverlapping. If $(X, T)$ is a cascade and if $\alpha$ is a finite closed nonoverlapping cover of $X$, then $\bigvee_{i=0}^{n-1} T^{-i} \alpha$ is also a finite closed nonoverlapping cover for any positive

Received by the editors May 6, 1969. 
integer $n$. Here, it is essential that $T$ be a homeomorphism, and it is for this reason that we restrict our attention at first to cascades.

Proposition 1. Let $\alpha$ be a finite closed nonoverlapping cover of $X$. Then $N(\alpha)$ is the number of sets of $\alpha$ which have interior.

Proof. Let $\beta$ be the collection of sets in $\alpha$ which have interior, and let $W$ be the union of the sets in $\alpha$ which do not have interior. Then

$$
\bigcap_{A \in \beta}(X-A) \subset W .
$$

But $\bigcap_{A \in \beta}(X-A)$ is open, and $W$ has no interior, so $\bigcap_{A \in \beta}(X-A)$ must be empty, which means that $\beta$ is a cover of $X$. On the other hand, each set of $\beta$ is essential to $\alpha$, for if $A \in \beta$ were not essential, then $A \subset \bigcup_{B \in \alpha ; B \neq A} B$, so $A \subset \bigcup_{B \in \alpha ; B \neq A} B \cap A$, contradicting the fact that $A$ has interior. Hence $\beta$ is a subcover of $\alpha$ of minimal cardinality.

CoRollary 1. Let $\alpha$ and $\beta$ be finite closed nonoverlapping covers of $X$ and $Y$ respectively. Then $N(\alpha \times \beta)=N(\alpha) \cdot N(\beta)$. Hence, if $(X, T)$ and $(Y, S)$ are cascades, $h(\alpha \times \beta, T \times S)=h(\alpha, T)+h(\beta, S)$.

Proposition 2. Every finite closed cover of $X$ has a closed nonoverlapping shrinking.

Proof. Let $\alpha=\left\{A_{1}, \ldots, A_{q}\right\}$ be a closed cover. Define $B_{i}=A_{i}-\bigcup_{j<i} A_{j}^{0}$, $i=1, \ldots, q$, where $A_{j}^{0}$ denotes the interior of $A_{j}$. Then for $i<k, B_{i} \cap B_{k} \subset A_{i}-A_{i}^{0}$, and so $B=\left\{B_{1}, \ldots, B_{q}\right\}$ is nonoverlapping.

2. The theorem for cascades. We let $I$ denote the interval $[0,1]$ and we let $Z$ denote the set of integers and $Z^{+}$the set of nonnegative integers. For a positive integer $m$, we let $C_{m}$ denote the set of all bisequences $(\ldots x(-1), x(0), x(1), \ldots)$ of points in $I^{m}$, i.e., $C_{m}=\left(I^{m}\right)^{z}$. We let $C_{m}$ have the product topology and we define $\sigma_{m}: C_{m} \rightarrow C_{m}$ by the rule

$$
\sigma_{m}(x)(n)=x(n+1) \text { for } x \in C_{m}, n \in Z .
$$

We let $\pi_{m}: C_{m} \rightarrow I^{m}$ be the projection

$$
\pi_{m}(x)=x(0) \text { for } x \in C_{m} .
$$

We shall use the following theorem from dimension theory:

THEOREM. For every open cover of $I^{m}$ there is a finite closed refinement of order $\leqq m+1$.

If $(X, T)$ is a cascade, then it is easily seen that, for positive integers $p$ and $n$ and a cover $\alpha$ of $X$,

$$
\bigvee_{i=-p}^{p+n} T^{-i} \alpha \subset \bigvee_{j=0}^{n} T^{-j}\left(\bigvee_{i=-p}^{p} T^{-i} \alpha\right)
$$


It follows by a straightforward argument that

$$
h\left(\bigvee_{i=-p}^{p} T^{-i} \alpha, T\right)=h(\alpha, T)
$$

Lemma 1. Let $(X, T)$ and $(Y, S)$ be subcascades of $\left(C_{m}, \sigma_{m}\right)$. Then

$$
h(T)+h(S) \leqq h(T \times S)+2 \log (m+1) .
$$

Proof. Let $\alpha$ be an open cover of $C_{m}$. Because of the definition of the topology for $C_{m}$, we can refine $\alpha$ to a basic open cover of the form

$$
\alpha^{\prime}=\bigvee_{i=-p}^{p} \sigma_{m}^{-i} \pi_{m}^{-1}\left(\beta_{i}\right)
$$

where $p$ is a positive integer and the $\beta_{i}$ are open covers of $I^{m}$. Furthermore, taking $\beta=\bigvee_{i=-p}^{p} \beta_{i}$, we have

$$
\bigvee_{i=-p}^{p} \sigma_{m}^{-i} \pi_{m}^{-1}(\beta) \succ \alpha .
$$

Now by the above mentioned theorem in dimension theory, we can choose a finite closed cover $\gamma$ of $I^{m}$ of order $\leqq m+1$ which is a refinement of $\beta$. Letting $\beta_{1}=$ $\left\{\pi_{m}^{-1}(B) \cap X \mid B \in \beta\right\}$ and $\beta_{2}=\left\{\pi_{m}^{-1}(B) \cap Y \mid B \in \beta\right\}$, we can apply Proposition 2 to obtain shrinkings $\delta_{1}$ of $\beta_{1}$ and $\delta_{2}$ of $\beta_{2}$ which are closed nonoverlapping covers of $X$ and $Y$ respectively. Now $\delta_{1}$ and $\delta_{2}$ each have order $\leqq m+1$, so $\delta_{1} \times \delta_{2}$ has order $\leqq(m+1)^{2}$, and it follows from Proposition 2 of [3] that

$$
h\left(\delta_{1} \times \delta_{2}, T \times S\right) \leqq h(T \times S)+2 \log (m+1) .
$$

Now using Corollary 1 ,

$$
\begin{aligned}
h_{X}(\alpha, T)+h_{Y}(\alpha, S) & \leqq h\left(\bigvee_{i=-p}^{p} T^{-i} \beta_{1}, T\right)+h\left(\bigvee_{i=-p}^{p} S^{-i} \beta_{2}, S\right) \\
& =h\left(\beta_{1}, T\right)+h\left(\beta_{2}, S\right) \leqq h\left(\delta_{1}, T\right)+h\left(\delta_{2}, S\right) \\
& =h\left(\delta_{1} \times \delta_{2}, T \times S\right) \leqq h(T \times S)+2 \log (m+1)
\end{aligned}
$$

Now since $\alpha$ was an arbitrary open cover, the lemma is proved.

COROLlaRY 2. If $(X, T)$ and $(Y, S)$ are subcascades of $\left(C_{m}, \sigma_{m}\right)$, then

$$
h(T)+h(S)=h(T \times S) .
$$

Proof. Let $n$ be a positive integer. Then $\left(X, T^{n}\right)$ and $\left(Y, S^{n}\right)$ are subcascades of $\left(C_{m}, \sigma_{m}^{n}\right)$. But it can be shown, as in Proposition 3 of [3], that $\left(C_{m}, \sigma_{m}^{n}\right)$ is isomorphic to $\left(C_{m n}, \sigma_{m n}\right)$, so that from the lemma, $h\left(T^{n}\right)+h\left(S^{n}\right) \leqq h\left(T^{n} \times S^{n}\right)+2 \log (m n+1)$. Now, using Theorem 2 of [1], we have

$$
\begin{aligned}
h(T)+h(S)-h(T \times S) & =(1 / n)\left(h\left(T^{n}\right)+h\left(S^{n}\right)-h\left((T \times S)^{n}\right)\right) \\
& \leqq(1 / n) \cdot 2 \log (m n+1) .
\end{aligned}
$$


We now let $n$ tend to infinity and observe that $(1 / n) \cdot 2 \log (m n+1)$ tends to zero, giving $h(T)+h(S) \leqq h(T \times S)$. Since the reverse inequality was proved in [1], we are done.

If $(X, T)$ is a cascade and $\phi$ is a homomorphism from $(X, T)$ into some $\left(C_{m}, \sigma_{m}\right)$, we say that $\phi$ is a representation of $(X, T)$, and we let $T_{\phi}$ denote the restriction of $\sigma_{m}$ to $\phi(X)$. We let $R^{\prime}(X, T)$ denote the set of all representations of $(X, T)$. The following proposition is completely analogous to Theorem 2 of [3], and the proof will be omitted.

Proposition 3. $h(T)=\sup \left\{h\left(T_{\phi}\right) \mid \phi \in R^{\prime}(X, T)\right\}$.

Lemma 2. If $(X, T)$ and $(Y, S)$ are cascades, then $h(T \times S)=h(T)+h(S)$.

Proof. Let $\varepsilon>0$. Then by the above proposition, we can choose $\phi \in R^{\prime}(X, T)$ and $\psi \in R^{\prime}(Y, S)$ such that $h(T) \leqq h\left(T_{\phi}\right)+\varepsilon / 2$ and $h(T) \leqq h\left(S_{\psi}\right)+\varepsilon / 2$. It follows from Corollary 2 that

$$
h(T)+h(S) \leqq h\left(T_{\phi}\right)+h\left(S_{\psi}\right)+\varepsilon=h\left(T_{\phi} \times S_{\psi}\right)+\varepsilon .
$$

However, the cascade $\left(\phi(X) \times \phi(Y), T_{\phi} \times S_{\psi}\right)$ is a homomorphic image of the cascade $(X \times Y, T \times S)$, so $h(T)+h(S) \leqq h(T \times S)+\varepsilon$. Hence, $h(T)+h(S) \leqq h(T \times S)$, and since the reverse inequality is known, the lemma is proved.

3. A theorem on inverse limits. In this section we assume that there is given a directed set $\mathscr{I}$, a system of flows $\left\{\left(X_{i}, T_{i}\right)\right\}_{i \in \mathscr{I}}$, and a consistent collection of homomorphisms $\lambda_{i j}:\left(X_{i}, T_{i}\right) \rightarrow\left(X_{j}, T_{j}\right)$ for $i, j \in \mathscr{I}, i \geqq j$.

The inverse limit of this system is defined to be the flow $(X, T)$, where

$$
X=\left\{x=\left(x_{i}\right)_{i \in \mathscr{I}} \in \prod_{i=\mathscr{I}} X_{i} \mid \lambda_{j k}\left(x_{j}\right)=x_{k} \text { for } j, k \in \mathscr{I}, j \geqq k\right\},
$$

and $T: X \rightarrow X$ is given by

$$
(T(x))_{i}=T_{i}\left(x_{i}\right) \text { for } x \in X, i \in \mathscr{I} .
$$

$X$ is given the topology induced from the product topology on $\prod_{i \in \mathscr{I}} X_{i}$. We let $\lambda_{i}: X \rightarrow X_{i}$ be the coordinate projections restricted to $X$.

THEOREM 1. $h(T) \leqq \sup _{i \in \mathscr{I}} h\left(T_{i}\right)$, and if the bonding maps $\lambda_{i j}$ are all surjective, then $h(T)=\lim _{i \in \mathscr{I}} h\left(T_{i}\right)$.

Before proving Theorem 1, we note that the requirement that the bonding maps be surjective is necessary for the equality $h(T)=\lim _{i \in S} h\left(T_{i}\right)$. For if we let $X_{i}=$ $\left\{x \in C_{1} \mid x(n) \leqq 1 / i\right.$ for all $\left.n \in Z\right\}$, and $T_{i}=\left.\sigma_{1}\right|_{x_{i}}$, for $i \in Z^{+}$, and if we let the $\lambda_{i j}$ be the inclusion maps, then it can be easily seen that the inverse limit flow $(X, T)$ is the trivial one-point flow. On the other hand, it follows from work in [1] that $h\left(T_{i}\right)=\infty$ for $i \in Z^{+}$.

To prove Theorem 1, we need a lemma about inverse limits of compact Hausdorff spaces which has nothing to do with flows. 
LEMmA 3. For each open cover $\alpha$ of $X$, there is an index $k \in \mathscr{I}$ and an open cover $\beta$ of $X_{k}$ such that $\lambda_{k}^{-1}(\beta)>\alpha$.

Proof. Because of the compactness of $X$ and the definition of the product topology, we can choose a basic refinement of $\alpha$ of the form:

$$
\gamma=\lambda_{i_{1}}^{-1}\left(\gamma_{1}\right) \vee \lambda_{i_{2}}^{-1}\left(\gamma_{2}\right) \vee \cdots \vee \lambda_{i_{q}}^{-1}\left(\gamma_{q}\right),
$$

where $\left\{i_{1}, \ldots, i_{q}\right\}$ is a finite subset of $\mathscr{I}$, and $\gamma_{1}, \ldots, \gamma_{q}$ are open covers of $X_{i_{1}}, \ldots, X_{i_{q}}$ respectively. We now let $k=\max \left\{i_{1}, \ldots, i_{q}\right\}$ and note that because of the consistency of the $\lambda_{i j}$, we have

$$
\lambda_{i_{r}}^{-1}\left(\gamma_{r}\right)=\lambda_{k}^{-1}\left(\lambda_{k i_{r}}^{-1}\left(\gamma_{r}\right)\right) \text { for } r=1, \ldots, q .
$$

Now letting $\beta=\bigvee_{r=1}^{q} \lambda_{k i_{r}}^{-1}\left(\gamma_{r}\right)$, we have $\lambda_{k}^{-1}(\beta)=\gamma \succ \alpha$, and we are done.

Proof of Theorem 1. For each open cover $\alpha$ of $X$, we let $k$ and $\beta$ be as in the lemma. It follows from Property 7 of [1], that $h\left(\lambda_{k}^{-1}(\beta), T\right) \leqq h\left(\beta, T_{k}\right)$. Hence,

$$
h(\alpha, T) \leqq h\left(\lambda_{k}^{-1}(\beta), T\right) \leqq h\left(\beta, T_{k}\right) \leqq h\left(T_{k}\right) \leqq \sup _{i \in \mathscr{J}} h\left(T_{i}\right) .
$$

Since $\alpha$ was arbitrary, we have $h(T) \leqq \sup _{i \in \mathscr{I}} h\left(T_{i}\right)$. Now if the $\lambda_{i j}$ are assumed to be surjective, then the $\lambda_{i}$ are also surjective, and it follows that $h(T) \geqq h\left(T_{i}\right)$ for all $i \in \mathscr{I}$. It also follows that, for $i \geqq j, h\left(T_{i}\right) \geqq h\left(T_{j}\right)$, so that $h(T)=\lim _{i \in \mathcal{S}} h\left(T_{i}\right)$.

4. The general product theorem. In this section we generalize Lemma 2, using a method introduced in [2] for constructing a cascade from a given flow.

If $(X, T)$ is a flow, we let $\left(X^{*}, T^{*}\right)$ be the inverse limit of $\left\{\left(X_{i}, T_{i}\right)\right\}_{i \in Z^{+}}$, where each $\left(X_{i}, T_{i}\right)=(X, T)$, and the bonding maps are given by $\lambda_{i j}=T^{i-j}$, for $i \geqq j$. Theorem 1 yields $h\left(T^{*}\right) \leqq h(T)$, and in fact, we shall show $h\left(T^{*}\right)=h(T)$. We note that $\left(X^{*}, T^{*}\right)$ is a cascade; for the inverse of $T^{*}$ is the shift, $\sigma$, defined by $(\sigma(x))_{i}=x_{i+1}$, for $x \in X^{*}, i \in Z^{+}$.

We define

$$
\tilde{X}=\bigcap_{n \in Z^{+}} T^{n}(X) \text { and } \tilde{T}=T \mid \tilde{X} .
$$

It is evident that $\tilde{T}(\tilde{X}) \subset \tilde{X}$, and we claim that, in fact, $\tilde{T}(\tilde{X})=\tilde{X}$. For let $x \in \tilde{X}$. Then for each $n \in Z^{+}, T^{-1}(x) \cap T^{n}(X) \neq \varnothing$, and it follows from the compactness of $T^{-1}(x)$ that

so that $\tilde{T}$ maps $\tilde{X}$ onto $X$.

$$
T^{-1}(x) \cap \tilde{X}=T^{-1}(x) \cap \bigcap_{n=1}^{\infty} T^{n}(X) \neq \varnothing,
$$

Proposition 4. If $U$ is an open set containing $\tilde{X}$, then there is some $k \in Z^{+}$such that $T^{k}(X) \subset U$.

Proof. This follows from the compactness of $X$, and the fact that

$$
\left\{X-T^{n}(X) \mid n \in Z\right\} \cup\{U\}
$$

is an open cover of $X$. 
Proposition 5. $h(\tilde{T})=h(T)$.

Proof. Let $\alpha$ be an open cover of $X$ and let $\varepsilon>0$. From the definition of $h_{\tilde{X}}(\alpha, \tilde{T})$, we can choose $m \in Z^{+}$so large that

$$
\frac{1}{m} \log N_{\tilde{X}}\left(\bigvee_{i=0}^{m-1} T^{-i} \alpha\right)<h_{\tilde{X}}(\alpha, \tilde{T})+\varepsilon
$$

Let $\beta$ be a subcover of $\bigvee_{\imath=0}^{m-1} T^{-i} \alpha$ of minimal cardinality relative to $\tilde{X}$. By Proposition 4, we can choose a $k$ so large that $T^{-k}(\beta)$ covers $X$. Hence, using Property 4 and Property 7 of [1], we have, for any $n \in Z^{+}$,

$$
\begin{aligned}
N\left(\bigvee_{i=0}^{m n-1} T^{-i} \alpha\right) & \leqq N\left(\bigvee_{i=0}^{m n+k-1} T^{-i} \alpha\right) \\
& =N\left(\left(\bigvee_{i=0}^{k-1} T^{-i} \alpha\right) \vee T^{-k}\left(\bigvee_{i=0}^{m-1} T^{-i} \alpha\right) \vee T^{-m} T^{-k}\left(\bigvee_{i=0}^{m-1} T^{-i} \alpha\right)\right. \\
\left.\vee \cdots \vee T^{-m(n+1)} T^{-k}\left(\bigvee_{i=0}^{m-1} T^{-i} \alpha\right)\right) & \leqq N\left(\bigvee_{i=0}^{k-1} T^{-i} \alpha\right) \cdot\left(N\left(T^{-k}\left(\bigvee_{i=0}^{m-1} T^{-i} \alpha\right)\right)\right)^{n} \leqq N\left(\bigvee_{i=0}^{k-1} T^{-i} \alpha\right) \cdot\left(N_{\tilde{X}}(\beta)\right)^{n} .
\end{aligned}
$$

Hence,

$$
\begin{aligned}
\frac{1}{m n} \log N\left(\bigvee_{i=0}^{m n-1} T^{-i} \alpha\right) & \leqq \frac{1}{m n} \log N\left(\bigvee_{i=0}^{k-1} T^{-i} \alpha\right)+\frac{1}{m} \log N_{\tilde{X}}(\beta) \\
& <\frac{1}{m n} \log N\left(\bigvee_{i=0}^{k-1} T^{-i} \alpha\right)+h_{\tilde{X}}(\alpha, \tilde{T})+\varepsilon .
\end{aligned}
$$

Now, letting $n$ tend to infinity, we note that $(1 / m n) \log N\left(\bigvee_{i=0}^{k-1} T^{-i} \alpha\right)$ tends to zero, so we have $h(\alpha, T) \leqq h_{\tilde{X}}(\alpha, \tilde{T})+\varepsilon \leqq h(\tilde{T})+\varepsilon$. Since $\varepsilon$ and $\alpha$ were arbitrary, we have $h(T) \leqq h(\tilde{T})$. But the reverse inequality follows from Theorem 4 of [1], so the proposition is proved.

Proposition 6. $h\left(T^{*}\right)=h(T)$.

Proof. We simply note that $\left(\tilde{X}^{*}, \tilde{T}^{*}\right)=\left(X^{*}, T^{*}\right)$ so that, by Proposition 5 and the second part of Theorem 1 , we have $h(T)=h(\tilde{T})=h\left(\tilde{T}^{*}\right)=h\left(T^{*}\right)$.

THEOREM 2. $h(T \times S)=h(T)+h(S)$.

Proof. We first note that $\left(X^{*} \times Y^{*}, T^{*} \times S^{*}\right)$ is canonically isomorphic to $\left((X \times Y)^{*},(T \times S)^{*}\right)$. Hence, by Proposition 6 and Lemma 2,

$$
h(T \times S)=h\left((T \times S)^{*}\right)=h\left(T^{*} \times S^{*}\right)=h\left(T^{*}\right)+h\left(S^{*}\right)=h(T)+h(S) .
$$

We can generalize even further:

THEOREM 3. Let $\left\{\left(X_{k}, T_{k}\right)\right\}_{k \in \mathscr{X}}$ be an arbitrary collection of flows. Let $X=\prod_{k \in \mathscr{X}} X_{k}$, and let $T: X \rightarrow X$ be defined by

$$
(T(x))_{k}=T_{k}\left(x_{k}\right), \quad x \in X, k \in \mathscr{K} .
$$

Then $h(T)=\sum_{k \in \mathscr{K}} h\left(T_{k}\right)$. 
Proof. We let $\mathscr{I}$ be the collection of all finite subsets of $\mathscr{K}$, ordered by inclusion. We let $\left(Y_{i}, S_{i}\right)=\prod_{k \in i}\left(X_{k}, T_{k}\right)$, and we let $\lambda_{i j}$ be the natural projections. It is straightforward to show that $(X, T)$ is isomorphic to the inverse limit of the system $\left\{\left(Y_{i}, S_{i}\right)\right\}_{i \in \mathscr{F}}$. We now note that, by Theorem $2, h\left(S_{i}\right)=\sum_{k \in i} h\left(T_{k}\right)$, and so, by the second part of Theorem 1,

$$
h(T)=\lim _{i \in \mathscr{I}} h\left(S_{i}\right)=\lim _{i \in \mathscr{J}} \sum_{k \in i} h\left(T_{k}\right)=\sum_{k \in \mathscr{X}} h\left(T_{k}\right) .
$$

5. An example. In this section we construct a cascade which has topological entropy equal to zero, and which has a finite closed cover having entropy equal to $\log 2$.

The cascade will be a subcascade of the sequence cascade $\left(C_{1}, \sigma_{1}\right)$ defined in $\$ 2$. For each positive integer $m$, we define $X_{m}$ to be the set of all bisequences $x=$ $(\cdots x(-1), x(0), x(1), \cdots) \in C_{1}$ satisfying the requirement that there are no more than $m$ integers $i$ such that $\left|x(i)-\frac{1}{2}\right|>1 / m$. It can be checked that $X_{m}$ is closed and $\sigma_{1}$-invariant. We now define

$$
X=\bigcap_{m=1}^{\infty} X_{m}, \quad T=\left.\sigma_{1}\right|_{x} \text { and } \pi=\left.\pi_{1}\right|_{x} .
$$

To show that $h(T)=0$, it will suffice to show that $h\left(\pi^{-1}(\alpha), T\right)=0$ for open covers $\alpha$ of $I$, because every open cover of $X$ has a refinement of the form $\bigvee_{i=-q}^{q} T^{-i} \pi^{-1} \alpha$. Let us write $\alpha=\left\{V_{1}, \ldots, V_{t}\right\}$, and $U_{i}=\pi^{-1}\left(V_{i}\right)$ for $i=1, \ldots, t$. We can choose $V_{r} \in \alpha$ such that $\frac{1}{2} \in V_{r}$, and we can choose $m$ so large that the interval $\left[\frac{1}{2}-1 / m, \frac{1}{2}+1 / m\right]$ is contained in $V_{r}$. For a fixed integer $n$ larger than $m$, we let $\mathscr{A}$ be the set of all subsets of $\{0,1, \ldots, n-1\}$ with exactly $m$ members, so that $\mathscr{A}$ contains $n ! / m !(n-m) !$ sets. For $A \in \mathscr{A}$, we let $A^{\prime}$ denote the complement of $A$ in $\{0,1, \ldots, n-1\}$. For a function $c$ from a set $A \in \mathscr{A}$ into the set $\{1, \ldots, t\}$, we define

$$
W_{c}=\left(\bigcap_{i \in A^{\prime}} T^{-i} U_{r}\right) \cap\left(\bigcap_{i \in A} T^{-i} U_{c(i)}\right),
$$

which is a member of $\bigvee_{i=0}^{n-1} T^{-i} \pi^{-1}(\alpha)$. We let

$$
\gamma=\left\{W_{c} \mid A \in \mathscr{A} \text { and } c: A \rightarrow\{1, \ldots, t\}\right\},
$$

and we observe that $\gamma$ has no more than $(n ! / m !(n-m) !) p^{m}$ members, hence no more than $n^{m} p^{m}$ members. Next, we claim that $\gamma$ covers $X$. Let $x \in X$. Then $x \in X_{m}$, so there is a set $A \in \mathscr{A}$ such that $\left|x(i)-\frac{1}{2}\right| \leqq 1 / m$ for all $i \in A^{\prime}$. Thus, for $i \in A^{\prime}$, $\pi\left(T^{i} x\right)=\sigma^{i}(x)(0)=x(i) \in V_{r}$, so that $T^{i} x \in U_{r}$, and hence $x \in \bigcap_{i \in A^{\prime}} T^{-i} U_{r}$. Now, for $i \in A$, we choose $c(i)$ so that $T^{i} x \in U_{c(i)}$, and it follows that $x \in W_{c}$. This shows that $\gamma$ covers $X$, so that we have a subcover of $\bigvee_{i=0}^{n-1} T^{-i} \pi^{-1}(\alpha)$ with no more than $n^{m} p^{m}$ members, and we have $N\left(\bigvee_{i=0}^{n-1} T^{-i} \pi^{-1}(\alpha)\right) \leqq n^{m} p^{m}$. Hence,

$$
\frac{1}{n} \log N\left(\bigvee_{i=0}^{n-1} T^{-i} \pi^{-1}(\alpha)\right) \leqq \frac{1}{n} \log p^{m} n^{m}
$$

and letting $n$ tend to infinity, we obtain $h\left(\pi^{-1}(\alpha), T\right)=0$. Hence, the topological entropy of $T$ is zero. 
We now define $F_{0}=\left\{x \in X: x(0) \in\left[0, \frac{1}{2}\right]\right\}$ and $F_{1}=\left\{x \in X: x(0) \in\left[\frac{1}{2}, 1\right]\right\}$, and let $\beta=\left\{F_{0}, F_{1}\right\}$. We claim that $h(\beta, T)=\log 2$. Let $n$ be a fixed positive integer. For each function $b$ from $\{0, \ldots, n-1\}$ into $\{0,1\}$, we let

$$
G_{b}=F_{b(0)} \cap T^{-1} F_{b(1)} \cap \cdots \cap T^{-n+1} F_{n-1} \in \bigvee_{i=0}^{n-1} T^{-i} \beta
$$

and we let $x_{b}$ be the point of $X$ defined by the rule

$$
\begin{aligned}
x_{b} & =\frac{1}{2}-(-1)^{b(i)} / n & & \text { for } i=0,1, \ldots, n-1, \\
& =0 & & \text { for } i<0 \text { or } i \geqq n .
\end{aligned}
$$

Then it can be shown that $x_{b} \in G_{b}$ and that $x_{b} \notin G_{b^{\prime}}$ whenever $b^{\prime} \neq b$. Thus, for each $b:\{0, \ldots, n-1\} \rightarrow\{0,1\}, G_{b}$ is an essential member of the cover $\bigvee_{i=0}^{n-1} T^{-i} \beta$, so that $N\left(\bigvee_{i=0}^{n-1} T^{-i} \beta\right)=2^{n}$. Hence,

$$
\frac{1}{n} \log N\left(\bigvee_{i=0}^{n-1} T^{-i} \beta\right)=\log 2
$$

Since $n$ was arbitrary, $h(\beta, T)=\log 2$.

\section{REFERENCES}

1. R. Adler, A. Konheim and M. McAndrew, Topological entropy, Trans. Amer. Math. Soc. 114 (1965), 309-319. MR 30 \#5291.

2. M. Eisenburg, Expansive transformation semigroups of endomorphisms, Fund. Math. 59 (1966), 313-321. MR 34 \#3562.

3. L. W. Goodwyn, Topological entropy bounds measure-theoretic entropy, Proc. Amer. Math. Soc. 23 (1969), 679-688. MR 40 \#299.

UNIVERSITY OF KENTUCKY,

LeXINGTON, KeNTUCKY 40506 